\title{
In-space Assembly Capability Assessment for Potential Human Exploration and Science Applications
}

\author{
Sharon A. Jefferies ${ }^{1}$, Christopher A. Jones ${ }^{2}$, Dale C. Arney ${ }^{3}$, Frederic H. Stillwagen ${ }^{4}$, Patrick R. Chai ${ }^{5}$, Craig D. \\ Hutchinson $^{6}$, Matthew A. Stafford ${ }^{7}$, Robert W. Moses ${ }^{8}$, James A. Dempsey ${ }^{9}$ \\ NASA Langley Research Center, Hampton, VA, 23681, USA \\ Erica M. Rodgers ${ }^{10}$ \\ NASA Headquarters, Washington, D.C., 20024, USA \\ Henry H. Kwan ${ }^{11}$ \\ Georgia Institute of Technology, Atlanta, GA, 30332, USA \\ and \\ Sean P. Downs ${ }^{12}$ \\ University of Colorado Boulder, Boulder, CO, 80309, USA
}

\begin{abstract}
Human missions to Mars present several major challenges that must be overcome, including delivering multiple large mass and volume elements, keeping the crew safe and productive, meeting cost constraints, and ensuring a sustainable campaign. Traditional methods for executing human Mars missions minimize or eliminate in-space assembly, which provides a narrow range of options for addressing these challenges and limits the types of missions that can be performed. This paper discusses recent work to evaluate how the inclusion of in-space assembly in space mission architectural concepts could provide novel solutions to address these challenges by increasing operational flexibility, robustness, risk reduction, crew health and safety, and sustainability. A hierarchical framework is presented to characterize assembly strategies, assembly tasks, and the required capabilities to assemble mission systems in space. The framework is used to identify general mission system design considerations and assembly system characteristics by assembly strategy. These general approaches are then applied to identify potential in-space assembly applications to address each challenge. Through this process, several focus areas were identified where applications of in-space assembly could affect multiple challenges. Each focus area was developed to identify functions, potential assembly solutions and operations, key architectural trades, and potential considerations and implications of implementation. This paper helps to identify key areas to investigate were potentially significant gains in
\end{abstract}

\footnotetext{
${ }^{1}$ Aerospace Engineer, Space Mission Analysis Branch, MS 462, 1 N. Dryden Street, Hampton VA, Senior Member.

${ }^{2}$ Aerospace Engineer, Space Mission Analysis Branch, MS 462, 1 N. Dryden Street, Hampton VA, Member.

${ }^{3}$ Aerospace Engineer, Space Mission Analysis Branch, MS 462, 1 N. Dryden Street, Hampton VA, Member.

${ }^{4}$ Communications and Tracking Systems Security Engineer, Space Mission Analysis Branch, MS 462, 1 N. Dryden Street, Hampton VA, Non-Member.

${ }^{5}$ Aerospace Engineer, Space Mission Analysis Branch, MS 462, 1 N. Dryden Street, Hampton VA, Member.

${ }^{6}$ Aerospace Engineer, Space Mission Analysis Branch, MS 462, 1 N. Dryden Street, Hampton VA, Senior Member.

${ }^{7}$ Electronics Engineer, Systems Integration \& Test Branch, MS 424, 21 Langley Boulevard, Hampton VA, Senior Member.

${ }^{8}$ Aerospace Engineer, Atmospheric Flight and Entry Systems Branch, MS 482, 1 N. Dryden Street, Hampton VA, Senior Member.

${ }^{9}$ Aerospace Engineer, Space Mission Analysis Branch, MS 462, 1 North Dryden Street, Hampton VA, Member.

${ }^{10}$ Aerospace Engineer, Office of Chief Technologist, 300 E St SW, Washington, DC, Senior Member.

${ }^{11}$ NASA Intern, Space Mission Analysis Branch, MS 462, 1 North Dryden Street, Hampton VA, Member.

12 NASA Intern, Space Mission Analysis Branch, MS 462, 1 North Dryden Street, Hampton VA, Non-Member.
} 
addressing the challenges with human missions to Mars may be realized, and creates a foundation on which to further develop and analyze in-space assembly concepts and assembly-based architectures.

\section{Introduction}

U UMANITY continues to reach into space with increasingly more ambitious missions to expand our knowledge of the universe and extend our presence throughout the solar system. Missions to Mars are a long held goal for human exploration. The traditional approach for executing these missions requires the launch of integrated, fullyfunctioning systems that become operational with little more than a series of deployments. However, most Mars mission architectures, especially those proposing long-duration surface stays or a sustained presence require hundreds to thousands of tonnes of mass in low Earth orbit or cislunar space to enable the missions. ${ }^{1-3}$ The transportation stages, landers, and other systems required by those architectures can exceed the launch capabilities of current and expected future launch vehicles. Thus, it can be difficult to design fully integrated systems that meet the requirements for a Mars mission within the constraints of launch vehicle capabilities.

In-space assembly (ISA) offers approaches to addressing this challenge. While current large payloads face design constraints imposed by launch vehicle fairing dimensions and payload capacities, in-space assembly could enable payloads to be launched piecemeal and integrated in space. In addition to addressing challenges with mass and volume limitations of launch vehicles, in-space assembly provides the potential to address many other challenges relating to operational flexibility, robustness, risk reduction, crew health and safety, and sustainability.

In this study, in-space assembly ranges from the mating of two elements to create an operational mission system, to the assembly of an element from a large and diverse collection of parts used to assemble components, subsystems, and modules. By this definition, the International Space Station represents the largest current example of in-space assembly: many mostly or fully functional elements were launched and joined together using a combination of crewed and robotic assembly. Future applications of in-space assembly may significantly enhance or enable the ability to meet human space exploration goals. The selected in-space assembly capabilities may also have crossapplicability for a range of science and robotic missions.

To that end, this paper examines in-space assembly strategies and capabilities, both with respect to human missions to Mars as well as broader classes of in-space applications. This analysis serves two purposes: 1) to understand the state of the art and capability needs for in-space assembly as it relates to potential human and science applications, and 2) to inform future in-space assembly development decisions with respect to recently proposed human Mars mission architectures.

To meet the first purpose, this paper examines a range of challenges for human exploration of Mars that could be met by in-space assembly. Examining several potential exploration and science applications of in-space assembly yields a framework of assembly strategies, tasks, and capabilities that could be applied to meet the exploration challenges.

To meet the second purpose, this paper describes potential applications within several "focus areas" for the use of in-space assembly to human Mars missions. These focus areas, arising from a synthesis of the assembly framework and the previously identified challenges, represent stages or regions within a Mars mission where opportunities for in-space assembly to improve the architecture or campaign can be traded. These focus areas serve as a starting point for future architecting of in-space assembly-enabled Mars campaigns.

\section{Challenges with Human Exploration of Mars}

Human exploration of Mars, a long-held goal for human space flight, captures the imagination with visions of long-term habitation and colonization. However, missions to Mars that propose long-duration surface stays or a sustained presence face many challenges affecting cost, risk, execution, and sustainability. ${ }^{4}$ Turning these Mars mission dreams into reality will require innovative solutions that can address the many challenges that currently limit our ability to realize such ambitious missions.

One of the most readily apparent challenges relates to the sheer amount and size of the equipment necessary to safely execute Mars missions and provide for the crew. Traditional mission approaches typically call for the launch of fully integrated mission systems that are operational upon reaching orbit with minimum assembly. However, the mass and size of Mars mission-class systems, particularly transportation stages and landers loaded with payload, can exceed the launch capabilities of current and planned launch vehicles. ${ }^{1,5,6}$ Recent Mars architectures, such as Mars DRA 5.0 and the Evolvable Mars Campaign, incorporate a minimal level of assembly using docking for aggregation 
of propulsion stages to other elements, but each element is otherwise designed to be fully operable upon reaching orbit.

In addition to the challenges with mass and volume limitations of launch vehicles, several other challenges with Mars missions must be addressed. The study team, working with a group of subject matter experts on system, architecture, and campaign development for Mars missions and on in-space assembly, identified multiple challenges and assessed each for the potential for ISA to provide a viable solution. The list of those challenges with potential ISA applicability is in Table 1.

Table 1. Challenges with human exploration of Mars

\begin{tabular}{|c|c|c|}
\hline \multicolumn{3}{|c|}{ Human Exploration Challenges } \\
\hline $\begin{array}{l}\text { Overall affordability } \\
\text { - Availability of systems over long } \\
\text { lifetimes } \\
\text {. Reusability \& sustainability } \\
\text { - Dormancy } \\
\text { - Staging of elements } \\
\text { - Aggregation of elements } \\
\text { - Launch vehicle cost } \\
\text { - Launch vehicle reliability } \\
\text { - Launch vehicle packaging }\end{array}$ & $\begin{array}{l}\text { Crew health, safety, well-being } \\
\text { Radiation mitigation } \\
\text { Microgravity health effects } \\
\text { Adequate crew volume } \\
\text { Safe haven } \\
\text { Logistics } \\
\text { Micrometeoroids and orbital } \\
\text { debris protection } \\
\text { Repurposing / recycling } \\
\text { Mission ops at Mars (time delay) }\end{array}$ & $\begin{array}{l}\text { Data rates and availability of } \\
\text { communication } \\
\text {. Lander packaging } \\
\text {. Entry, descent, and landing } \\
\text { Civil engineering / site } \\
\text { preparation } \\
\text {. Surface hazards } \\
\text { Power generation / energy storage } \\
\text { on surface } \\
\cdot \text { Planetary protection } \\
\text {. Ascent from Mars }\end{array}$ \\
\hline
\end{tabular}

In addressing these challenges, in-space assembly has the potential to provide increased flexibility in architecture and launch manifesting options, more robustness to launch failures, reduced burden on ground testing and handling facilities, and opportunities for "pay-as-you-go" architectures that can spread out development and operational costs over time. Additionally, it is anticipated that the ability to assemble systems at the destination can lead to packaging efficiencies and reduce aero-capture, entry, and descent risks to the payloads. Furthermore, the availability and use of assembly assets and infrastructure over multiple missions allow for amortization of system costs over a longer period of time and open the possibility of using the assets to support system servicing and upgrades.

\section{On In-Space Assembly}

As technologies have advanced over the years, in-space assembly is becoming an increasingly viable option for supporting or enabling space missions. While human crews can be used to perform some assembly operations, not all assemblies will be suitable for intra-vehicular activity, and extravehicular activity (EVA) operations are risky and inherently limited, both in duration and number, by availability of the crew and resources. Robotic capabilities and autonomous systems are advancing to the point where robotic in-space assembly is becoming feasible without unnecessary risk to EVA crew or extensive oversight from human operators.

In order to better understand where and how ISA might be applied to address the challenges identified above, the study team examined a wide range of potential exploration and science mission system applications to identify how each could be broken down for assembly at different levels, what tasks would be performed, and what capabilities would be required. These decompositions were informed by several recent and historical ISA-related papers and studies. ${ }^{7-11}$ The results of these evaluations contributed to development of a common nomenclature and a framework of assembly strategies, tasks, and capabilities that can be applied to meet the exploration challenges.

The framework provides a means of relating and communicating the strategies one can take to perform in-space assembly for a particular mission system, the design considerations for the mission system that enable it to be assembled, the types of operational tasks that would be performed to carry out that assembly strategy, and the performance parameters that drive the assembly capability needs and assembly asset implementation options. The functional capabilities can support multiple tasks, and a given operational task may be called for within different strategies. The primary differences for tasks and capabilities that fall within different strategies are the level of complexity and the performance requirements on the capability.

\section{A. Mission Applications}

Mission systems are the vehicles, spacecraft, or other elements (or combinations thereof) that are assembled to conduct some mission. They are distinct from any "assembly agents", which are systems that perform assembly 
tasks on the mission system, and from "assembly infrastructure", which are facilities in space that support assembly by providing berthing and storage for the mission system and assembly agents. A mission system could exist without in-space assembly, as is traditionally the case, although some missions systems may be too large or complex to launch as an integrated system on current and planned launch vehicles. The full list of identified mission systems is in Table 2. Eight mission systems, in boldface in the table below, were selected for the initial decomposition that was used to identify the assembly strategies, tasks, and capabilities that are discussed in below and explore the relations among them.

Table 2. Mission system candidates for assembly

\begin{tabular}{|c|c|}
\hline 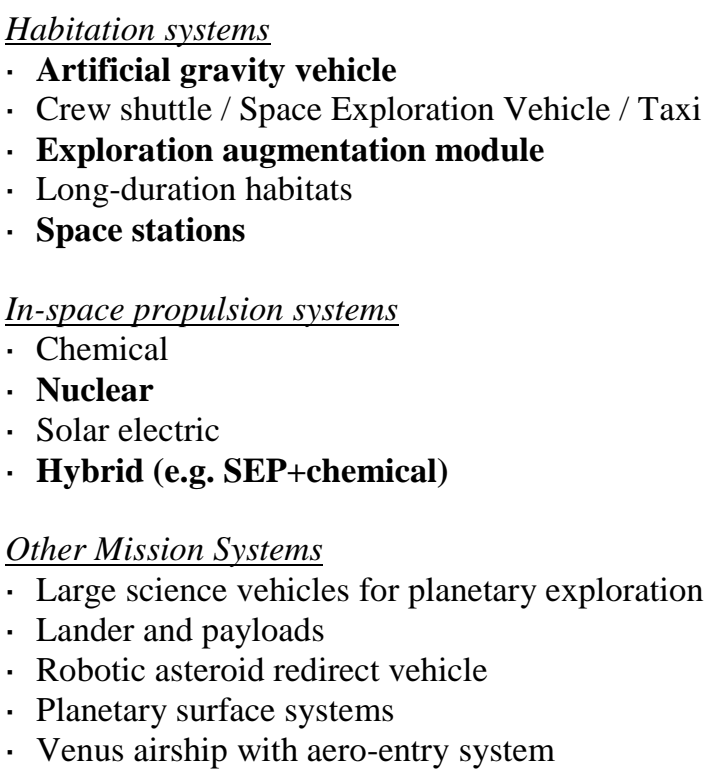 & $\begin{array}{l}\text { Mission Platforms } \\
\text {. Commercial GEO communications platform } \\
\text {. Deep-space communications platforms } \\
\text {. In-space power stations (in-space to surface, in- } \\
\text { space to in-space e.g. laser propulsion) } \\
\text {. Large-aperture observatory (e.g. high definition } \\
\text { space telescope) } \\
\text {. Persistent platform (permanent backbone with } \\
\text { systems and instruments changed out) } \\
\text { Infrastructure } \\
\text {. Fuel depot } \\
\text {. Space dock } \\
\text { - In-space hangar (may be similar to dock or large } \\
\text { habitat, has enclosed volume) }\end{array}$ \\
\hline
\end{tabular}

\section{B. Assembly Strategies}

Assembly strategies are the broad approaches that can be taken to assemble a spacecraft in space. They represent the differing degrees of assembly complexity that can be employed as well as the design philosophy for the mission system itself. As assembly complexity increases, additional assembly tasks may be performed, and assembly agents and infrastructure will need to provide more capabilities and meet more demanding functional specifications. Mission systems must be designed intentionally to take best advantage of in-space assembly capabilities, and the designers will need to consider the assembly capabilities that are expected to be available throughout the lifetime of the system.

Five assembly strategies were identified that categorize the degree of assembly complexity, ranging from no assembly to assembly from parts. Figure 1 provides a graphical representation that exemplifies each strategy, and then they are further described, from simplest to most complex, in the following list. Table 3 provides additional details relating mission system designs to the assembly strategies.

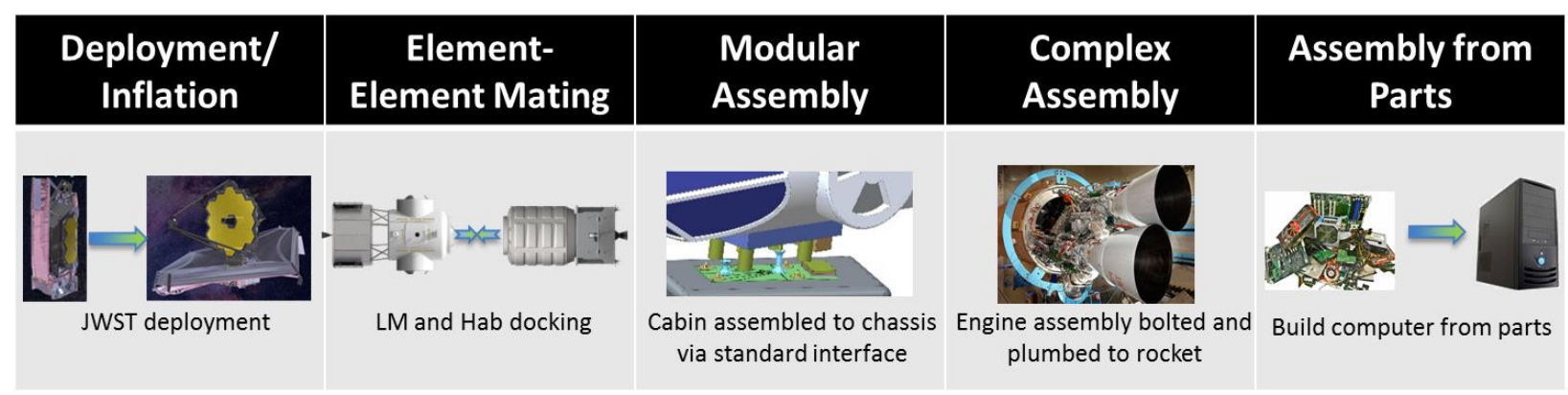

Figure 1. Example representations of assembly strategies 
1) Deployment/inflation - The element is delivered as a single piece requiring no joining or connection to other elements or components. Deployment or inflation of stowed components may be necessary, but no physically distinct components of the spacecraft exist. This strategy thus involves no assembly, but may use robotic assistance that requires capabilities similar to those needed for assembly tasks.

2) Element-to-element mating - Two or more highly integrated elements are delivered to space, where they are assembled with a minimum of complexity. The complexity is similar to basic docking or berthing: most, if not all, connections are handled by the mating interface. Mating interfaces tend to have design features that provide greater tolerance/margin for positioning and alignment, which reduces the functional needs on any assembly agents or automated systems.

3) Modular assembly of components - An element is assembled from physically distinct components that are designed to be connected with little physical interaction to connect the interfaces. The complexity is analogous to plugging a laptop into a docking station or sliding instrument modules into a rack with preseated connections. Joining and restraining methods are simplified and designed to reduce alignment error and have greater tolerance. Access to the interfaces is typically easy and open.

4) Complex assembly of components - An element is assembled from physically distinct components that require significant manipulation and interaction to connect. The complexity is analogous to installing a new hard drive in your desktop computer, or having to make all the power, data, and fluid connections to the instruments/subsystems as a separate step in the process. Access may be more restricted, or may involve particular sequencing, and physical/structural connections may be more complex (e.g. installing bolts or twisting connectors).

5) Assembly from parts - An element is assembled from physically distinct components that are themselves assembled from many parts; multiple stages of assembly between the initial set of components and final element are thus required. The complexity is analogous to building up a computer from parts (motherboard, processor, heat sink, fan, audio and video cards, power supply, etc.), building trusses from structural members, or installing mirror segments and alignment mechanisms onto a structural frame.

Assembly of a particular mission system may incorporate a mixture of these strategies for different parts of the spacecraft, and in combination as parts and subassemblies could be built into subsystems and elements that are then joined with other components or mated to additional elements. For example, assembly operations primarily using element-to-element mating or modular assembly with simplified, plug-and-play-type connections, may also require some more complex joining operations (e.g. for fluid, thermal, and RF connections). Additionally, assembly may be sequenced so that individual elements are assembled via modular or more complex assembly, and then mated to other elements. At the lowest level, parts that are built up into subassemblies can then be assembled into subsystems and elements via modular and/or complex assembly. Thus, it is possible for a given assembly to incorporate operations from all of the strategies.

This study assessed in-space assembly separately from manufacturing and fabrication. Manufacturing and fabrication are not ISA strategies, but can be an extension of the above strategies, adding the use of in situ or delivered resources to the manufacture of parts or components, which then would feed the above strategies. Manufacturing and fabrication are alternative means of providing the parts and components that would be used for assembly and are highly synergistic with the "assembly from parts" and "complex assembly of components" strategies; examples include building up solar array cells from semiconducting metal; assembling a rotary joint from nuts, bolts, and gears; and extruding truss members and fabricating nodes to build structures.

\section{Assembly Tasks}

Assembly tasks are the operations performed in the process of assembling a mission system. In the course of an assembly, the same task (or sequence of tasks) may be performed multiple times, at varying degrees of precision or complexity. The assembly tasks can be sequenced together to create an assembly concept of operations (con-ops) that would suffice to describe the operational steps required to assemble a mission system. For each assembly task, there exist one or more associated capabilities that combine to provide the functions needed to execute the task. Eleven assembly tasks were identified that serve as the building blocks for describing an assembly con-ops: 
Table 3. Mission system design considerations by assembly strategy.

\begin{tabular}{|c|c|c|}
\hline Strategy & Representative Component Interactions & Mission System Design Needs \\
\hline $\begin{array}{l}\text { Element - } \\
\text { Element } \\
\text { Mating }\end{array}$ & $\begin{array}{l}\text { Habitat and propulsion bus } \\
\text { Multiple habitat elements } \\
\text { Bus and integrated communications tower } \\
\text { Lander to an integrated payload pallet }\end{array}$ & $\begin{array}{l}\text { Pre-integrated elements } \\
\cdot \text { Standard mating interfaces } \\
\text { - Functional distribution across elements }\end{array}$ \\
\hline $\begin{array}{l}\text { Modular } \\
\text { Assembly }\end{array}$ & $\begin{array}{l}\text { Tank and thruster modules to a structural } \\
\text { backbone for a propulsion bus } \\
\text { Instrument modules to a persistent platform } \\
\text { Mirror assembly to deployable sunshield and to } \\
\text { bus } \\
\text { Robotics and component storage to an assembly } \\
\text { platform/space dock } \\
\text { Solar array module to bus, electronics boxes }\end{array}$ & $\begin{array}{l}\text { - Modular, integrated components } \\
\text { Standard interfaces and connectors } \\
\cdot \text { Planned upgradeability } \\
\text { - Design for human/robotic assembly \& } \\
\text { repair }\end{array}$ \\
\hline $\begin{array}{l}\text { Complex } \\
\text { Assembly }\end{array}$ & $\begin{array}{l}\text { Solar panels/ antennae to masts, etc. } \\
\text { Connecting integrated mirror/antenna modules to } \\
\text { build larger apertures } \\
\text { Connection of fluid couplings } \\
\text { Routing of cables through structure }\end{array}$ & $\begin{array}{l}\text { Design for limited toolset } \\
\text { Standard components } \\
\cdot \text { Assembly sequence } \\
\text { Preplanned cable and fluid line routes } \\
\text { - Design for human/robotic assembly \& } \\
\text { repair }\end{array}$ \\
\hline $\begin{array}{l}\text { Assembly } \\
\text { from Parts }\end{array}$ & $\begin{array}{l}\text { Assembly of structures from struts and nodes. } \\
\text { Building large-aperture dishes from non-integrated } \\
\text { mirror/antenna panels, structural elements, and } \\
\text { electronics } \\
\text { Building solar array panels from solar cells and } \\
\text { structural components } \\
\text { Building subassemblies from constituent parts } \\
\text { (e.g. tanks, plumbing, thrusters, structure, and } \\
\text { other components to make a thruster module) }\end{array}$ & $\begin{array}{l}\text { - Build-up sequence } \\
\text { - Detailed assembly instructions } \\
\text { - Design for robotic assembly/ repair } \\
\text {. Part management }\end{array}$ \\
\hline
\end{tabular}

1) Maneuver in free flight - Includes rendezvous and approach movements between locations, primarily to move payloads, elements, and assembly assets to the assembly point via a free-flying assembly agent, and close proximity maneuvering of free-flying assembly agents around the assembly. All assembly strategies incorporate similar rendezvous and approach capabilities. Proximity operations may require different levels of capability depending on the assembly strategy. For instance, proximity operations in support of docking or berthing needs a similar level of safety and automation as currently used on ISS, while use of free-flying agents to support modular and more complex assembly operations will require a greater degree of situational awareness, autonomy, and precision control.

2) Dock or berth - Includes the connection of payloads (e.g. pallets) to infrastructure as an initial positioning prior to subsequent assembly tasks, as well as the connection of modules as part of element-to-element mating. The requirements for this task (including the precision and awareness required for any arm or other manipulator) are a function of the design of the elements or components to be mated.

3) Locate component - Includes finding a particular component in its stowage location or in the target area within the assembly location. It also includes acquiring and processing state knowledge of the assembly agent within the larger assembly space.

4) Acquire component - Includes accessing and manipulating restraints to release a component from storage and achieving positive control of the component. The requirements for this task depend upon the properties of the components being acquired; components of significantly different dimensions may require different manipulation and grapple capabilities. The situational awareness requirements may also vary depending on the sensitivity of the components to tolerances in position and force from the manipulator. 
5) Position component - Includes moving a component from its storage or holding location to the assembly location and into the correct orientation for joining the component to the assembly. If the assembly con-ops includes a free-flying assembly agent, the Maneuver in Free Flight task describes the gross movement of the assembly agent between locations, while this task describes the fine translation and rotation of the component.

6) Join components - Includes making the structural, power, data, fluid, and thermal connections between components. Depending on the nature of the interface and the complexity of assembly, this task may occur multiple times (requiring different capabilities in each instance) to completely join the components. For example, one end-effector may be required to physically join the components, while another is necessary to perform the connection of a fluid path.

7) Disjoin/disassemble - Includes undoing a previously joined assembly of components. This task may be required due to the particular sequence of assembly; for example, if an upgraded component is to be installed into a previously assembled element. Alternatively, this task may be required if verification and validation shows that an assembly was incorrectly joined.

8) Deploy components - Includes the deployment of a stowed component, whether the component selfdeploys or an assembly agent assists in the deployment. Whether this task exists in an assembly con-ops, and the requirements the task imposes, are a function of the nature of any deployed components in the mission system being assembled.

9) Secure/safe components - Includes putting a component into a state that can survive and endure nonassembly events or testing. In addition to the physical manipulation required to secure the component, this task also includes inspection of the component to verify the securing.

10) Verify assembly - Includes the steps taken to determine that the system was assembled properly and functions as required. This task can be performed at steps during the assembly con-ops, as well as after the completion of the assembly. The nature of the assembly being verified will determine the required capabilities.

11) Assess faults - Includes detecting and diagnosing faults in the assembly, and conveying that information to an overseer (either human or robotic). This does not include assessing faults within an assembly agent.

\section{Capabilities}

Capabilities

provide basic functionality needed to perform a given task to assemble a mission system. Multiple capabilities are typically needed to perform a given task. For example, positioning a component requires the situational awareness of where that component should go and the grappling and manipulation capability to actually move the component to that location.

The capabilities were identified through the examination of each of the above assembly tasks. The resulting list of capabilities (Table 4) has been organized into groups based on the types of function(s) being performed. Note that the capabilities listed here are at a

Table 4. Assembly capabilities grouped by function

\begin{tabular}{|c|c|}
\hline Rendezvous, Proximity & Control and Assembly \\
\hline Operations, and Mating & Automation Capabilities \\
\hline Capabilities & Provide Situational Awareness \\
\hline $\begin{array}{l}\text { Mate Elements (Berthing and } \\
\text { Docking) }\end{array}$ & $\begin{array}{l}\text { Provide Operator Situational } \\
\text { Awareness }\end{array}$ \\
\hline - Maneuver in Free Flight & Deployment Capabilities \\
\hline Assembly Capabilities & Deploy Structures \\
\hline - Join Fluid Connections & Deploy Inflatable Structure \\
\hline - Join Electrical/Data/RF & Verifying the Assembly \\
\hline Connections & Capabilities \\
\hline - Join Structural Connections & Verify by Testing \\
\hline - Perform Additive & Verify by Analysis \\
\hline Manufacturing & Verify by Inspection \\
\hline - Grapple Components & \\
\hline - Manipulate Components & \\
\hline
\end{tabular}
relatively high level, and would need to be broken down into sub-capabilities when developing more detailed con-ops. For example, Manipulate Components can be divided into coarse and fine manipulation, and Situational Awareness can include spatial awareness within the overall assembly (e.g., for path planning and proximity operations), ability to locate correct assembly parts and assembly location (i.e., close localization), and metrology. Determination of which subcapabilities are needed, and their associated performance parameters, will depend on both the mission system being assembled and the assembly strategy being used. Table 5 maps representative assembly capabilities and assembly asset needs to each assembly strategy. 
Table 5. Assembly agent and capability needs in relation to assembly strategy.

\begin{tabular}{|c|c|c|c|}
\hline $\begin{array}{l}\text { Assembly } \\
\text { Strategy }\end{array}$ & $\begin{array}{l}\text { Key Assembly } \\
\text { Features }\end{array}$ & $\begin{array}{c}\text { Significant } \\
\text { Capability Needs }\end{array}$ & $\begin{array}{l}\text { Potential Infrastructure/ } \\
\text { Assembly Agent Needs }\end{array}$ \\
\hline $\begin{array}{l}\text { Element- } \\
\text { Element } \\
\text { Mating }\end{array}$ & $\begin{array}{l}\text { Piloted/autonomous } \\
\text { rendezvous and } \\
\text { docking/berthing } \\
\text { - Robotic berthing arm }\end{array}$ & $\begin{array}{l}\text { Autonomous rendezvous and } \\
\text { docking }\end{array}$ & $\begin{array}{l}\text { Tug/bus to transport elements } \\
\text { Platform to secure element(s) } \\
\text { during assembly } \\
\text { - Robotics for berthing and more } \\
\text { complex connections } \\
\text { - Cameras for observation/ visual } \\
\text { inspection }\end{array}$ \\
\hline $\begin{array}{l}\text { Modular } \\
\text { Assembly }\end{array}$ & $\begin{array}{l}\text { Simplified assembly and } \\
\text { connections } \\
\text { - General-purpose robots } \\
\text { needing limited set of } \\
\text { end-effectors }\end{array}$ & $\begin{array}{l}\text { Situational awareness around } \\
\text { assembly and to find proper } \\
\text { assembly points } \\
\text { Handling and manipulation of } \\
\text { components into proper alignment } \\
\text { and position with moderate } \\
\text { tolerances }\end{array}$ & $\begin{array}{l}\text { Above }+ \\
\text { Platform to store components } \\
\text {. Agent that moves around } \\
\text { assembly site } \\
\text { - Additional inspection assets (e.g. } \\
\text { leak detection) } \\
\text { - Additional end-effectors and } \\
\text { assembly tools addressing } \\
\text { particular capability needs }\end{array}$ \\
\hline $\begin{array}{l}\text { Complex } \\
\text { Assembly }\end{array}$ & $\begin{array}{l}\text { - Manual (robotic or } \\
\text { human) connections } \\
\text { - Restricted workspace } \\
\text { - Increased dexterity } \\
\text { needed } \\
\text { - Pre-planned sequences / } \\
\text { robots that read } \\
\text { blueprints }\end{array}$ & $\begin{array}{l}\text { Joining power/ data/ RF/ thermal } \\
\text { connection } \\
\text {. Routing cables and connections } \\
\text { through areas with tight or limited } \\
\text { access } \\
\text {. Joining methods beyond force } \\
\text { application } \\
\text { - Precise autonomous manipulation } \\
\text { and alignment } \\
\text { - Situational awareness and ability } \\
\text { to access and navigate through } \\
\text { constrained locations } \\
\text { Verification/validation methods } \\
\text { suitable for assemblies built in } \\
\text { space }\end{array}$ & $\begin{array}{l}\text { Above }+ \\
\text { Additional end-effectors and } \\
\text { assembly tools addressing } \\
\text { particular capability needs } \\
\text { - Manipulators with more precise } \\
\text { motions, sensing, and situational } \\
\text { awareness } \\
\text { - More refined component storage } \\
\text { and preparation areas } \\
\text { - Equipment for acceptance testing } \\
\text { of assemblies }\end{array}$ \\
\hline $\begin{array}{l}\text { Assembly } \\
\text { from Parts }\end{array}$ & $\begin{array}{l}\text { Couples with in-space } \\
\text { manufacturing and in- } \\
\text { situ resource utilization } \\
\text { - Highest precision, } \\
\text { dexterity, \& situational } \\
\text { awareness } \\
\text { - Tightly scripted routines } \\
\text { - Specialized robots }\end{array}$ & $\begin{array}{l}\text { Higher degree of precision } \\
\text { manipulation and situational } \\
\text { awareness } \\
\text { · Higher demands on autonomy } \\
\text { · More intricate joining methods for } \\
\text { small scale components } \\
\text { - Autonomously access and } \\
\text { navigate constrained locations }\end{array}$ & $\begin{array}{l}\text { Above }+ \\
\text { Manipulators and end effectors } \\
\text { with very fine(millimeter or } \\
\text { better) precision } \\
\text { - Equipment for acceptance testing } \\
\text { of subassemblies } \\
\text { - Additional infrastructure for } \\
\text { "workbenches" }\end{array}$ \\
\hline
\end{tabular}


Many of these capabilities have been demonstrated to some extent on ISS or during missions like those servicing Hubble with either astronaut EVA; teleoperations; or highly planned, scripted, and controlled robotic operations. However, in order to automate these functions and reduce the human burden and risk, advancements are needed in each area. Rendezvous, proximity operations, and mating capabilities have been used extensively in the construction and general operation of ISS, but these operations have humans monitoring and often operating the systems. The robotics needed for the assembly capabilities of most mission systems are state of the art, but the automation and situational awareness capabilities of those robots need advancement. Deployment capabilities in space have limited scope and scale; the Bigelow Expandable Activity Module (BEAM) and the James Webb Space Telescope are the current state of the art. Larger or more exotic deployable structures (e.g. larger habitats, propellant tanks, solar arrays) would require additional advancement or need to leverage robotic assistance. Finally, the verification paradigm focuses on ground-integrated spacecraft, so advancements in testing, analysis, and inspection for in-space assembled structures will be needed.

\section{Application of In-Space Assembly to Human Exploration}

The framework discussed in Section III provides a means for defining potential applications of ISA, identifying different options in the trade space, and determining potential functional requirements for execution. With this better understanding of ISA and its applications, the human exploration challenges identified in Section II were evaluated to identify and define potential ISA solutions, including: implementation options within human Mars missions, potential benefits provided by ISA, and new implications or "costs" introduced by incorporating ISA. A brief synopsis of the considerations for each challenge is captured below.

1) Overall affordability - The challenge is the high costs for enabling and conducting human missions to Mars. NASA cannot afford to do all developments for a Mars surface mission simultaneously. This challenge applies to all aspects in a Mars mission, including architecture element development and testing, Earth-to-orbit launch, mission operations, etc. This challenge must be solved, as high costs may result in delays or program cancellation. The use of in-space assembly may allow for options that can reduce cost (e.g., use of less expensive launch vehicles and sequenced build-up in a "pay-as-you go" architecture). Inspace assembly is a viable option to meet this challenge.

2) Availability of systems over long lifetimes - The challenge is achieving the ability for systems to be functional over long (multi-year) durations while providing required functionality (e.g., power generation). This challenge applies to all elements that must remain operational for long durations, especially transEarth injection, ascent, surface architecture, habitats, etc. that are in continuous or intermittent use for years. This challenge must be solved, since many of these elements are involved in mission critical events. The typical approaches to solve or mitigate this challenge are by using spares and redundant subsystems. This challenge could be met with in-space assembly by incorporating modular designs so that systems can be disassembled and components replaced, or by adding components to augment performance (e.g., installing additional solar arrays). In-space assembly is a viable option for this challenge.

3) Reusability and sustainability - The challenges are increasing the efficiency with which mass is utilized in a Mars mission (for example through refueling, reconfiguration, and repurposing), and providing more costeffective approaches to providing mission capabilities. The first challenge is focused on amortizing mission system costs over long durations and facilitating continuing missions, while the second is focused on increasing the value proposition to incentivize investment in space. These challenge should be solved, but are not critical to mission success. This challenge could possibly be met with in-space assembly by incorporating modular spacecraft designs that can be launched on a variety of launch vehicles, reconfiguring or upgrading systems in space over time, disassembling systems and repurposing components after initial use is completed, and enabling reusable systems such as landers. Incorporating an assembly-based design philosophy can also open opportunities for commercial delivery and assembly services by providing a ready market, which builds the space economy and spreads the development costs over multiple providers. In-space assembly is a viable option for this challenge.

4) Dormancy - The challenge of dormancy of systems is the need for systems to be reliable and operational after long periods of non-use. This challenge applies to elements that must be pre-deployed months or years before use (for example a Mars surface habitat), which must survive in a challenging environment without 
constant supervision and maintenance. This challenge will likely have to be solved, as current architectures depend upon predeployment of cargo years in advance of its use. Some of the capabilities and design principles associated with in-space assembly, such as modular design, may help mitigate the challenge of dormancy. In-space assembly is a viable option for this challenge.

5) Staging of elements - The challenge is managing the pre-deployment of systems in Mars orbit and on the surface, and includes both the duration between deployment and use and ensuring systems are operational when needed for the mission. Many payloads, especially those delivered to the surface, have to be packaged for launch or landing in configurations that require some setup prior to use, due to launch vehicle or lander packaging constraints. Pre-deployed in-space propulsion stages for the return trip to Earth may also have to be mated. This challenge will need to be solved, because critical systems must be checked out and confirmed operational before the crew arrival. In-space assembly can help meet this challenge by enabling assembly and verification of staged systems to put them into an operational state. In-space assembly also provides greater flexibility in the design of mission systems, which can facilitate setup. Inspace assembly is a viable option for this challenge.

6) Aggregation of elements - The challenge is the need to bring many large, complex systems together to perform a Mars mission that cannot fit pre-integrated on a single launch vehicle. Aggregation can take place near Earth or near Mars, and applies to habitation, landing, and in-space transportation systems, as well as logistics or other payloads. Rendezvous, proximity operations, and docking or berthing of vehicles are fundamental operations for any crewed mission beyond low Earth orbit, but these put a heavy reliance and burden on navigational capabilities and performance/accuracy of the aggregated elements. Earth tracking services are not likely optimal for this challenge, so navigational aides have to be pre-positioned or "baked" into each element or payload. This challenge will need to be met, given the limitations of launch vehicles and in-space transportation stages. In-space assembly provides aggregation and docking of components, and modular design for assembly significantly expands the initial design space when formulating mission and campaign strategies. In-space assembly is a viable option for this challenge.

7) Launch vehicle cost - The challenge is to create a more sustainable and affordable launch capability. Launch vehicle costs ripple through the entire architecture. This challenge should be solved to allow for funding to be allocated to other system developments. In-space assembly would allow the architecture to be configured so that it can take advantage of lower cost commercial launch options and use commercial space development capabilities as they become available. In-space assembly provides flexibility to use different sized launch vehicles for different payloads, instead of a "one-size fits all" vehicle. In-space assembly is a viable option for this challenge.

8) Launch vehicle reliability - The challenge is the risk and impact of launch failure on a campaign. If every launch delivers a crucial element, then any failure is catastrophic. In addition, low launch rates lead to long times for launch vehicles to achieve maturity. This challenge applies primarily to payload launches, although crew launches could also benefit from increased launch vehicle maturity, and must be solved. Inspace assembly allows payloads to be divided for launch, which provides a greater number launch packages that drive an increased flight rate for launch vehicles. The greater number of launches results in reliability growth, thus decreasing the likelihood of launch failure. In addition, smaller payload launches can fly lower value, more readily replaceable parts, thus reducing the consequence of a specific launch failure. In-space assembly is a viable option for this challenge.

9) Launch vehicle packaging - The challenge is the difficulty or inability to fit large, fully-integrated systems for launch within the launch mass capacity and fairing dimensions of current or planned launch vehicles. This challenge applies to all mission and campaign scenarios that are heavily dependent on heavy-lift and large payload volumes as well as all mission and campaign scenarios where single-launch or singlecomponent failures present high risk. This challenge must be addressed for long-term sustainability and affordability, as well as increased robustness to failure relative to the state of the art. Currently, this challenge is not addressed except in some cases by commercial entities. In-space assembly could allow for modular designs and more efficient packaging that fit within a variety of LV and fairing options. In-space assembly is a viable option for this challenge. 
10) Crew health, safety, and well-being - The challenge is keeping the crew healthy (both physically and psychologically), safe, and content. The long durations and distances associated with Mars missions put constraints on the kinds of medical facilities that are available, and the confinement can have both physical and psychological effects. This challenge must be solved for crew in transit as well as on the surface. Inspace assembly has the potential to alleviate the detrimental effects by enabling systems that provide artificial gravity and by providing an opportunity to expand habitable volume, augment medical facilities, and improve communication capabilities. In-space assembly is a viable option for this challenge.

11) Radiation mitigation - The challenge is protecting crew from the negative health effects of galactic cosmic ray (GCR) and solar particle event (SPE) radiation. This challenge applies to all phases of a Mars mission, including cislunar, interplanetary, in Mars orbit, and on the surface of Mars. This challenge must be addressed to mitigate the long term impact on human crew, particularly if limits are found for the central nervous or cardiovascular systems. In-space assembly can be used to mitigate the effects of radiation through construction of large shields for habitats, or through the construction of more capable propulsion stages to enable faster transit. In-space assembly is a viable option for this challenge.

12) Microgravity health effects - The challenge is the physiological problems resulting from long-duration exposure of the crew to microgravity. This challenge applies to in-space transit and on the surface of Mars; however, the reduced gravity on a planet may not be as significant a risk. Microgravity creates crew health issues in bone health, muscle, and nuerovestibular adaptation to Mars environment. This challenge must be solved; Mars cannot be explored if the crew operations are impaired or they are at risk of injury. Artificial gravity could be used to solve this issue and lends itself well to in-space assembly. In-space assembly is a viable option for this challenge.

13) Adequate crew volume - The challenge is providing adequate habitable space for the crew to thrive physically and psychologically. Without adequate volume, the long durations in close quarters can produce physical and mental health threats that jeopardize the mission. This challenge must be solved, as the probability of loss of crew and mission is significantly impacted by the crew volume risks. In-space assembly permits larger habitable volumes to be assembled to address these risks. In-space assembly is a viable option for this challenge.

14) Safe haven - The challenge is providing protection for the crew from fire, decompression, malfunction, and other risks. Safe havens are typically provided by separate habitable volumes, which requires significant mass. This is a challenge that must be solved to mitigate risk to the crew. In-space assembly allows for modular construction and spreading of functionality and redundancy over multiple modules or parts of a single element. In-space assembly is a viable option for this challenge.

15) Logistics - The challenge is in meeting crew needs for consumables, food, and spare parts, as well as a standardized logistics delivery system. This challenge applies to launch and long-duration missions. The ability to repurpose and reuse logistics must be solved for sustainability. Aside from the basic docking or berthing of the logistics module to the spacecraft, in-space assembly provides the ability to repurpose, redistribute, and assemble logistics module on the surface (e.g. reconfigure fuel tanks and other logistics containers to assemble other structures on surface). This challenge requires in-space assembly.

16) Micrometeoroids and orbital debris (MMOD) protection - The challenge is the threat MMOD pose to spacecraft. This challenge applies to vehicle safety over long durations. This challenge must be solved for Low Earth Orbit (LEO) and anywhere the MMOD risk is moderate. Generally, the MMOD environment beyond LEO is benign, but the effect of an impact can be catastrophic. MMOD is currently being addressed by traditional shielding methods. In-space assembly can allow for construction of larger shields, installation of shielding after launch, or alternative shielding technologies. In-space assembly is a viable option for this challenge.

17) Repurposing and recycling - The challenge is reducing the mass requirements for a Mars campaign by effectively getting multiple uses out of certain systems and components. Achieving sustainability via repurposing and recycling will allow for lower sustaining costs for subsequent missions, as well as offering a means to mitigate risk. This challenge should be solved due to those benefits; however, it is not necessary 
as the demands of a campaign could be met without it. Capabilities associated with in-space assembly, such as the ability to disassemble systems, could support repurposing and recycling. In-space assembly is a viable option for this challenge.

18) Mission operations at Mars - The challenges include human and robotic mission operations at sites remote from Earth, communications back to Earth, and local navigation. Human and robotic mission operations at sites remote from Earth involve operations at locations resulting in significant time-delayed communication with Earth or that require independence from Earth, which influences required crew skill sets. Operations at Mars must also rely on a currently limited set of local assets for navigation and situational awareness. This challenge should be solved since there are higher risks associated with remote human operations. This challenge is currently being addressed through investments in autonomous systems technology. In-space assembly can provide solutions for this challenge by enabling construction or expansion of communications and navigation assets that provide improved coverage and data throughput. In-space assembly is a viable option for this challenge.

19) Data rates and availability of communication - The challenge is providing sufficient bandwidth and frequency of communication to ensure successful and safe operations on the way to, at, and returning from Mars. This includes accommodating the ability to communicate given the varying time latencies involved while traveling to and at Mars locations (up to 20 minutes one way). Communication methods and techniques have to accommodate these delays and continue to operate to support crew needs. Crewed missions will benefit from higher data rates than used for current science missions and will need the ability to communicate at any time with Earth when necessary (i.e. medical emergency). This challenge should be solved, as it will improve safety and increase scientific return. In-space assembly can provide for larger, more capable communication assets that increase bandwidth, coverage, and frequency of contact with Earth. In-space assembly is a viable option for this challenge.

20) Lander packaging - The challenge is the packaging of multiple manifests using a common descent stage design, while meeting launch vehicle fairing restrictions, protecting the payloads from thermal loads during entry, and maintaining a safe operating envelope throughout descent and landing. This challenge has generally been independent of launch mass; payload manifesting has been volume limited rather than mass limited. Significant previous packaging drivers have been ascent stage size and volume, and propellant volume for both ascent and descent stages. This challenge applies to all surface campaigns requiring one or more landings involving crew and a significant amount of surface architecture elements. This challenge must be solved for all campaigns that deliver large payloads to the surface. The typical solution has been to plan for the largest available launch fairing and to force the lander and element designs to fit, but satisfactory solutions cannot always be found that also meet EDL requirements. In-space assembly allows for payloads to be modularized or divided for safer, more efficient packaging for landing and then assembled on the surface. Some degree of surface assembly will be necessary for any human mission beyond "flag-and-footprints". This challenge requires in-space assembly.

21) Entry, descent, and landing (EDL) - The challenge is landing large payloads on the surface of Mars. Current Mars EDL concepts used for robotic science missions are mass limited to just under 1 metric ton. Human missions to the surface of Mars will require masses at least 1 order of magnitude larger. This challenge must be solved for human crews landing on Mars. Currently, integrated aeroentry device concepts that enable delivery of $\sim 25 \mathrm{t}$ to the surface are being developed, but have not been validated at this scale. In-space assembly could assemble large heat shields or augment retropropulsion capabilities in-situ, enabling larger landers to safely enter and land. Alternatively, surface assembly could enable the use of smaller landers that make use of more readily scalable EDL systems. In-space assembly is a viable option for this challenge.

22) Civil engineering and site preparation - The challenge is preparing the surface for landing, operations, and ascent. The natural terrain on Mars may not be suitable or adequate for surface missions; for example, there may be a risk of too much ejecta from landing impacting other elements of the surface architecture. This is a challenge that may need to be solved, depending on the nature of the surface layout and the geography of the local terrain. In-space assembly may allow for surface site preparation systems to be assembled and deployed. In-space assembly is a viable option for this challenge.

12

American Institute of Aeronautics and Astronautics 
23) Surface hazards - The challenge is the protection of crew and equipment from environmental hazards such as radiation, dust storms, temperature extremes, dust, and pluming/kick-up from landers, rovers, crew, and micro-meteoroids. This challenge applies to Mars surface stay and protection of equipment on the surface prior to crew arrival. This challenge must be solved, particularly for long stays of equipment or crew. Inspace assembly could provide the building of protective shields and berms, using materials on-surface or discarded equipment to erect protective shields. This challenge requires in-space assembly.

24) Power generation and energy storage on surface - The challenge is generating sufficient power to support crew missions. This challenge includes all phases of the mission: operations in cislunar space, interplanetary, in Mars orbit and on Mars surface. This challenge must be solved since significant quantities of power are required to support human Mars missions. In-space assembly could be leveraged through the deployment and assembly of large arrays in Mars orbit and on the surface. In-space assembly is a viable option for this challenge.

25) Planetary protection - The challenge is how to protect life on Mars and human life from each other, as well as prevent the contamination of the surface by humans. This challenge relates to habitation and crew activities on the surface. This challenge should be solved, since it is critical to protect humans and protocols established for planetary protection must be met. In-space assembly could facilitate construction of a dome for human habitation and isolation, as well as smaller separate isolation facilities to mitigate contamination. In-space assembly is a viable option for this challenge.

26) Mars ascent - The challenge is ensuring the crew leaves the surface and makes it safely into Mars orbit. This challenge must be solved for any mission with crew return. Current Mars Ascent Vehicle (MAV) concepts are constrained by the capabilities of launch, in-space transportation, and descent systems. Inspace assembly can either improve those systems, or be used to assemble or increase the capabilities of the ascent vehicle while on the surface. In-space assembly is a viable option for this challenge.

Each challenge was qualitatively assessed by a team of architecture and campaign subject matter experts for the anticipated impacts ISA would have on campaign cost, mission risk, and campaign value (e.g., through greater objective satisfaction or enabling of new science and exploration goals). Participants used a qualitative rating scale from 1 to 5 to score each challenge, where:

1: ISA would have a negligible positive impact (or a negative impact) on the benefit/risk/cost of the campaign

5: ISA would have a major positive impact on the benefit/risk/cost of the campaign

These assessments revealed the challenges with crew health, safety, and well-being, augmentations to radiation protection, launch packaging, EDL, surface site set-up, and Mars ascent had the highest likelihood of realizing major positive benefits from incorporating ISA. Specific results will be addressed in a future paper. Looking across the challenges revealed a significant overlap in the potential ISA solutions or mitigations. These ISA application areas, described in Section V, provide a focus for developing more specific ISA conceptual approaches.

\section{In-Space Assembly Focus Areas for Human Mars Missions}

The overlap observed when assessing ISA options for addressing the human exploration challenges led to the identification of a set of assembly focus areas that allow for the insertion of different assembly applications into the mission architecture. The focus areas concentrate on particular regions or systems within a mission where assembly

can occur and allow for more detailed assessments and trades on assembly operations options, the impacts of different assembly strategies, and the availability of assembly agent and infrastructure assets. The assembly focus areas include:

1) Earth-vicinity space yard

2) Modular, reusable Mars Transit Vehicle (includes habitation)

3) Mars orbit facility

4) Entry, descent, and landing

5) Mars surface assembly 
6) Communication and navigation infrastructure (in-space and surface)

Each of these focus areas were examined to identify the range of functions provided, assembly implementations and concepts of operations, key trades, and new considerations or problems introduced by the incorporation of ISA.

\section{A. Earth-vicinity space yard}

Function: The Earth-vicinity space yard is a facility in Earth's sphere of influence that is focused on assembly and checkout of deep space vehicles that could not fit inside of a single launch vehicle. This system would likely be located outside of the radiation belts and above the high density micrometeoroid and orbital debris (MMOD) regions. The potential functionality of this facility is vast and primarily depends on the result of trades for the assembled mission systems and the required level of assembly. The functions that this vehicle could perform include docking and berthing, construction of long structures (e.g., for artificial gravity or solar arrays), quality control (e.g., validation and verification) of the assembled systems, propellant storage and transfer, tug accommodations, logistics offloading and onboarding of fuel and supplies, dexterous manipulators, minimal additive manufacturing capability, tool and battery storage, crane like function (e.g., TALISMAN, ${ }^{12}$ and hovering inspection robots.

Solutions and Con-Ops: The Earth-vicinity space yard will primarily be robotic, with varying degees of autonomy and human interaction, e.g., teleoperation or crewed assembly. The simplest instantiations are the addition of robotic manipulators to an existing structure like a deep space gateway or a free-flying spacecraft with manipulators. A larger, more persistent facility could initially begin with a support bus and simple truss structure with robotics and a means to secure the system under assembly. Additional capabilities could be added over time through delivery of additional modules and support structure until a fully functional facility that provides a full range of assembly and support capabilities is completed. Support capabilities could include supply storage, servicing, verification and validation, and temporary berthing/staging of support vehicles such as tugs and tankers.

Key Trades: With the wide range of potential applications, a key trade centers on determining which use cases to implement. Other prominent trades include the size of launched payloads vs. the assembly operations and infrastructure needs, the degree and complexity of assembly performed, and the cost of assembly infrastructure against the number of missions and system types that infrastructure can assemble and other synergistic operations (i.e., servicing) it could support.

Considerations for Implementation: Utilizing an Earth-vicinity space yard for assembly operations introduces new architecture considerations and potential problems. Launching the assembly assets and performing assembly on the mission systems will take time that must be accounted for in mission planning and may delay mission dates. Additionally, incorporating assembly has more upfront cost, and the return on investment may not be realized until much later in the campaign.

\section{B. Mars Transit Vehicle}

Function: The Mars transit vehicle includes both the propulsion and habitation elements needed to transfer crew from Earth to Mars and back. The functions that this vehicle could perform include: sustaining the crew (both physically and psychologically), providing microgravity mitigation, providing radiation protection, providing auxiliary functions such as power and communications, and providing transit for crew from Earth to Mars and back. ${ }^{13,14,15}$

Solutions and Con-Ops: The habitat during deep space transit must protect the crew from physiological and psychological issues. Mitigating the negative health effects of microgravity through the use of artificial gravity, exercise, and large volumes may have feasible in-space assembly solutions. Radiation protection can be installed inside or around the exterior of the habitat to reduce exposure, and larger propulsion stages can be assembled to make the transit faster. MMOD protection can be installed on the habitat once in space to achieve greater standoff distances than can typically be achieved when installed for launch.

In-space assembly can address two main classes of habitat solutions: modular habitats and larger habitats constructed in space. The modular habitat links many smaller, functional habitation elements together, similar to the International Space Station (ISS). Modular habitats allow for redundancy, robustness against failure, and the potential for safe havens. The larger constructed habitat could take many forms, including expandable habitats, constructed rigid habitats, or a rigid truss with an inflatable bladder. Habitats can also be built from repurposed

14

American Institute of Aeronautics and Astronautics 
parts of launch vehicles (e.g. tanks). After launch, the interior can be outfitted and/or reconfigured on-orbit using the same types of capabilities as in-space assembly.

Propulsion stages can benefit greatly from in-space assembly, from reusing functional modules to salvaging tanks or engines from spent stages. Stages could be reusable and/or refuelable. More stages (and propellant) could provide a faster transit, reducing radiation exposure for the crew. Launch vehicle upper stages could be salvaged and reused for in-space transportation. For low-thrust propulsion, arrays or radiators can be constructed on orbit, mitigating issues with launch packaging and deployment.

Key Trades: Important trades for the Mars transit habitat focus on the structural design, which includes the size of the modules, how much of the system should be integrated or built on orbit, the amount of modularity versus the combination of monolithic and inflatable modules, and whether to mount habitat components internally or externally. Disaggregating parts of the habitat to use as a taxi or excursion vehicle to go to more locations is another trade key that focuses on the structural design.

Trades for the propulsion stage include whether to assemble a single large propulsion stage or aggregate multiple smaller propulsion stages that can separate off as necessary during transit, and whether to assemble faster, lighter transportation stage vs. a system that is slower and heavier. The use of reusable vs. disposable elements must also be traded for both the habitat and propulsion stage.

Considerations for Implementation: Using in-space assembly for preparation of the Mars transit vehicle introduces new architecture considerations and potential problems. As habitats increase in size, the ability of the propulsion system to deliver the payload in the desired timeframe may be exceeded, thus requiring a more powerful transportation stage. Additionally, inefficiencies are introduced as the number of habitation modules increases and their sizes diminish, and the risk of loss of a module from launch failure increases.

\section{Mars Orbit Facility}

Function: The Mars Orbital Facility is placed in Mars orbit to support inbound and outbound crew and cargo deliveries with Earth, as well as provide transition services for delivery to and from the surface and moons of Mars. It is primarily used to facilitate or enable mission operations, as opposed to serving as an assembly point for a new system. The functions that can be performed at the Mars Orbital Facility include refueling, component replacement and upgrade, aggregation/disaggregation of payloads and systems, processing and quarantine of samples, system checkout, communications/navigations support, and crew transfer support.

Solutions and Con-Ops: The Mars orbit facility will be mostly robotic and autonomous, although it could also operate with varying degrees of human interaction when crew are in the vicinity of Mars. The orbital facility can be used to receive crew, vehicles, and payloads from Earth, prepare landers for descent, receive and process crew and payloads for return to Earth, and service and refurbish reusable vehicles (e.g. landers and tugs) between missions. Orbital assembly at Mars opens several architecture and operational opportunities that can address multiple challenges. Decisions for which assembly capabilities and operations to provide in Mars orbit are tightly coupled with the Mars transit vehicle and EDL decisions.

In-space assembly at Mars can be as simple as transferring payloads from an Earth-Mars transfer vehicle to a lander or the mating of two predeployed propulsion stages, which would not require, but could be facilitated by, an orbital facility. A facility with some robotic arms and docking/berthing ports can support staging for systems (e.g., reducing those systems' orbital maintenance requirements), payload handling, and a variety of assembly functions. Adding features such as fuel tankage, lighting, and imaging systems can expand capabilities to include refueling and inspection. By including a pressurized tunnel or module, the facility can support crew transfer between Earth-Mars transportation stages or tugs and the lander or ascent vehicle, and could potentially serve as a safe haven. The Mars orbital facility is an enabler for reusable systems; allowing for refueling, replacement of expended components (e.g. landing gear), refurbishment, and upgrades to systems between missions. The facility can also host science payloads and comm/nav assets, both of which can be expanded or changed out over time.

Key Trades: Important trades for the Mars orbital facility include: where to locate the facility; how much assembly to perform in Mars orbit vs. at Earth or on the surface; when and how to build up assembly capabilities over time; whether to provide a habitable volume to support crew; additional capabilities and services that would be provided (e.g. servicing, refueling, logistics storage, comm/nav relay, etc.); and level of autonomy. 
Considerations for Implementation: Performing assembly operations at a Mars orbital facility introduces new architecture considerations and potential problems. These include delivery of assembly assets and infrastructure to Mars orbit, resultant implications of facility location on the architecture and mission operations, and the high degree of autonomy needed to enable operations given large time delays with Earth.

\section{Entry, Descent, and Landing}

Function: Entry, descent, and landing are critical functions for delivering crew and payloads to the surface. ${ }^{16,17}$ In-space assembly can address the challenges of entry, descent, and landing using two approaches. The first approach uses the assembly of a large heat shield in space, which then lands large masses on the surface. This approach enables delivery of larger monolithic payloads that will require minimal or no further assembly on the surface. The second approach divides surface sytems and packages the components onto smaller landers, which can utilize smaller EDL systems, for delivery and assembly on the surface. This enables EDL systems with less demanding design requirements, but places the burden on the surface assembly infrastructure and technologies.

Solutions and Con-Ops: The EDL approach depends on multiple architecture-level factors. The launch vehicle delivery mass and volume are the primary drivers for the EDL system configuration and payload delivery capability. From the surface architecture, the Mars ascent vehicle typically drives the minimum lander size, but the habitat, power system, and pressurized rovers can also set that constraint in some architectures. The specific EDL system configuration could also impact the viability of a given approach. Propulsive entry, deployable heat shields, and mid-lift-to-drag (mid-L/D) configurations as well as reusability, are all options in the EDL trade space that would impact the selected approach.

The first approach uses a large EDL system that is assembled in space. Pallets and components would be launched to the assembly location where the large heat shield would be assembled. A large sky crane descent system could also be assembled that fits behind the assembled heat shield. In addition to enabling delivery of fully integrated systems to the surface, larger landers also allow for more total mass to be delivered at one time, thus reducing the total number of landers required.

The second approach utilizes small landers and relies on surface assembly of the delivered systems. Given current EDL system designs, the largest payload delivery to the surface is on the order of 25 tonnes. ${ }^{1}$ Use of smaller landers can reduce design requirements on the EDL system, at the expense of more landers and surface assembly assets on the surface. Depending on lander size, multiple landers may be able to be launched together or delivered to Mars by the in-space transfer stage. This approach also includes utilization of sufficiently small landers that can be launched direct to Mars from Earth, reducing the need for a dedicated in-space transportation stage.

The two EDL approaches provide alternatives to address several challenges, including delivery of payloads to the surface, robustness to loss, and sustainability. Implementation of an EDL approach also ripples back to address challenges with launching payloads from Earth. These solutions open up possibilities such as reusable landers or arbitrarily large entry systems (and therefore larger payloads than could be achieved without assembly).

Key Trades: The primary trades for EDL are how big to make the lander and associated EDL system and how much assembly is performed either in space (approach 1) versus on the surface (approach 2). A related trade is whether to use reusable vs. disposable landers. Trades for approach 1 also include the costs of delivering larger payload masses to the surface vs. the costs associated with component delivery and in-space assembly required to build the EDL system and assembly the lander, EDL system, and payload together, how large to make the assembled EDL system, and where in space (Earth or Mars) the assembly occurs. For approach 2, an additional trade includes minimizing the division of elements to ease functionality vs. reassembling elements on the surface that can be landed using smaller EDL systems.

Considerations for Implementation: Performing assembly operations for EDL systems introduces new architecture considerations and potential problems. These include the need for additional infrastructure at Earth and/or Mars to support assembly and payload transfers, the scalability of different EDL concepts, and the need to autonomously locate and navigate to distributed landers and return payloads to the base assembly and use.

\section{E. Mars Surface Assembly}

Function: The Mars surface yard is not limited to a centralized location, but also includes use of mobile assembly agents distributed where needed across the surface to support assembly needs (e.g. for power systems). The functions that the surface yard could perform include: unloading, deploying, and assembling elements; providing habitation; providing radiation protection; providing sustainable power systems; providing in-situ 
resource utilization systems; performing civil engineering operations; providing mobility; performing repairs, maintenance, upgrades, expansions, repurposing, etc.; and providing waste management (burying sewage, trash handling, recycling center). The range of assembly functions required will depend on the architecture and assembly strategy employed. Because critical components of the base will need to be established prior to crew arrival, autonomy will be a key capability. ${ }^{18}$

Solutions and Con-Ops: A major component for Mars surface assembly is centered on safety. Several aspects of the surface base must be completed before crew arrival. ISA opens opportunities to address safety concerns by allowing the Mars surface architecture to be put set up, checked out, and verified operational prior to crew arrival. In addition, assembly provides a more sustainable path for human exploration by providing capabilities that enable reusability, site autonomy, and expansion. Architectures without some capability for assembly only allow for "flag and footprint"-type architectures.

Initial assembly operations may rely on only a few mobile assembly agents and cargo manipulation devices. As surface site build-up continues, assembly operations could expand to include more capable assembly assets and an increase in civil engineering capabilities. For architectures that deliver smaller payloads that will require assembly on the surface, centralized assembly infrastructure could be established to provide greater control over quality control, parts storage, and process management. The architecture and EDL approach will factor heavily in the assembly capabilities required on the surface.

The opportunities for in-space assembly with respect to the Mars surface yard could also require certain capabilities in surface elements, depending on architecture and assembly strategies chosen. For example, precision landing would be required and the degree of precision would depend on how distributed or centralized the surface yard is. The habitat design could either be separated into functional elements that are linked together or be flatpacked with pre-fabrication. A crane or similar system would provide capabilities to off-load elements from landers as well as load ascent vehicles. The time-delay from Earth to Mars and prior to crew arrival operations would require semi- or fully autonomous robotics. These robotic operations would be required for providing mobility to facilitate connections between modules, transport materials or moving bulk regolith, and providing dexterity for assembly operations.

Key Trades: The primary trade for the Mars surface yard is the level of assembly that occurs (e.g., connecting pre-integrated modules vs. building systems from modular components and prefabricated parts vs. building in-situ from raw materials), which drives the size of the payloads that need to be delivered and is tightly coupled with EDL trades and decisions. A related campaign trade is when to introduce more complex assembly capabilities over time to facilitate a shift from Earth dependence to Earth independence. Additional trades include: degree of human interaction required for assembly (from human performed to fully autonomous); degree of centralization for landed delivery of payloads (from single pad to widespread); and degree of commonality for modules and robots vs. specific designs.

Considerations for Implementation: Performing assembly operations at the surface yard introduces new architecture considerations and potential problems. These include the level of autonomy required, how much of the surface base has to be established before crew arrive, cost of development and delivery of surface assembly assets, assembly in a dusty environment, and surface infrastructure needs to locate and navigate to landers. Additional questions include when to transfer from Earth's manufacturing capability to Mars manufacturing and whether operations that rely on autonomy during setup will ever need to hand off those operations to humans after crew arrival.

\section{F. Communication and Navigation Infrastructure}

Function: The assembly of large orbital and surface communications and navigation aid (comm/nav) assets facilitates high data-rate return, near continuous communications coverage with Earth or Mars exploration assets, and navigation signaling support. All missions require a minimum level of comm/nav capability at Mars and at Earth, and as the number of missions and concurrent operations increase, comm/nav capabilities and capacity need to expand. Furthermore, by adding more than the minimum level of comm/nav capability, new or expanded scientific and crewed mission options may be enabled. In addition to providing added capability to support mission operations and return of scientific data for both crewed and uncrewed missions, greater communications and navigation capabilities will be needed to facilitate autonomous operations and assembly. 
Solutions and Con-Ops: Communications and navigational aids that are orbiting Mars, or on the surface, have to be able to support the selected Mars architecture to ensure mission success. Initial communications can likely be provided by the Mars transit vehicles and orbiting satellites, however, needs will change as the surface base grows. The comm/nav infrastructure in the Mars system can be augmented to provide the required communication and navigation support for elements that are being placed in Mars orbit, descending to or ascending from the Mars surface, or operating on the surface. Augmented comm/nav capabilities also enable precise navigation and location knowledge to support complex assembly operations. The expanded capability can be provided through delivery of new assets or by using in-space assembly to build additional capability into comm/nav systems already at Mars. Inspace assembly could benefit communications and navigation assets by enabling the modular build-up of capability as the exploration needs expand (i.e. "pay-as-you-go"). This includes adding power as well comm/nav equipment to support higher data transmission rates.

Key Trades: Key trades include determining the sizing and performance needs on the communication and navigation assets to support mission needs, and determining what communications/navigation capability to provide up front vs. build up over time. A related trade is whether to build capacity over time through addition of assets or using assembly to expand existing assets. An additional trade includes determining orbital vs. surface communications and navigation functional allocation and infrastructure needs.

Considerations for Implementation: Performing assembly operations for comm/nav systems introduces new architecture considerations and potential problems. Assembly operations are enabled by communications and navigation capabilities, so planning and development for assembly operations and asset capabilities must take into account available comm/nav support and need for expanded comm/nav capabilities. Increasing the ability to quickly transmit large amounts of data from Mars to Earth will require a corresponding increase of capability in Earthvicinity to receive the transmission. Additional communication infrastructure on the ground may also be required.

\section{Observations}

In-space assembly can help address the major challenges of Mars exploration (e.g. mission risk and safety) through broadening the trade space available for a Mars architecture or campaign, resulting in much greater mission flexibility over an architecture without in-space assembly. This broadened trade space is highly coupled, linking decisions across the campaign that the mission architect would otherwise make separately or sequentially. The inspace assembly infrastructure that is built up in these architectures is available for many missions, making the inclusion of in-space assembly in a Mars architecture a campaign-level decision.

In-space assembly broadens the architectural trade space, providing more mission flexibility and enabling new architecture options. By dividing elements into smaller components at launch, the usable suite of launch vehicles expands to encompass both SLS and commercial launch vehicles. This allows flexibility on recovering from failure, accelerating schedules, and potentially reducing launch cost, while also providing opportunities for greater commercial and international participation. In-space assembly creates an opportunity to reallocate payloads to other launch vehicles, minimizing schedule and costs impacts, and can also affect the evolution of launch vehicle development. For instance, the need for larger versions of the SLS can be reevaluated, because if the 8.4-meter fairing suffices for all foreseeable mission elements, then the development of a 10-meter fairing could be postponed or eliminated. Also, the options for placing surface systems on Mars expands with the potential of small landers with surface assembly, in-space assembly of large landers, and even the possibility of reusable Mars landers. The methodology introduced in this paper helps with the definition of the available trade space, and the different assembly strategies can be applied across the various mission systems in a given architecture.

In an architecture utilizing in-space assembly, there are significant interrelations among options in different parts of the trade space. Some architecture decisions dictate particular options while eliminating others, while in other cases, a given decision forces a series of decisions across the architecture. For example, assembling large payloads at Earth that must then transfer to Mars leads to a need to also develop or assemble more capable transportation systems. Another example involves assembling surface elements at Mars versus launching or assembling large surface elements in space. Postponing assembly to the surface of Mars in order to stay within landed mass limitations of the EDL system(s) offers some utility but with a price, as assembly agents will also need to be landed to conduct the surface assembly tasks. This approach (using surface assembly instead of in-space assembly) allows the investment in surface assembly capabilities to be weighed against alternative EDL options.

Finally, in-space assembly builds infrastructure that can be used across multiple missions, types of missions, or campaigns. Incorporating assembly agents and developing assembly capabilities have upfront costs, but these costs 
can be amortized over the operational life of the system and over the various missions that infrastructure would support. Therefore, assembly agents should be selected that can provide capabilities used to assemble a range of missions systems over a long lifetime in order to maximize value and justify the upfront costs. Certain capabilities stand out as having broad applicability across a majority of assembly tasks. While the specific sub-capabilities and associated performance parameters (precision, dexterity, accuracy, etc.) required of these capabilities will vary based on the task and assembly strategy, any advances in these broadly-applicable capabilities are expected to provide benefits to a wide range of assembly activities.

\section{Future Work}

The work to date has included a preliminary assessment of potential assembly applications in the five in-space assembly focus areas, and has focused on identifying benefits and costs of implementing different options and understanding the interrelation of assembly decisions

Further work will examine each of these areas to develop and assess specific assembly approaches, with a view towards generating representative architectures for human Mars missions that incorporate differing degrees of assembly and assembly complexity. These representative architectures will be used to define system concepts for assembly agents and infrastructure needed to enable the architecture. Campaigns built from these architectures will be quantitatively compared against more traditional, minimal-assembly campaigns to better define potential benefits and costs of the assembly-based architectures.

\section{Summary}

Future human space exploration missions will require large, complex systems. Due to the challenging requirements to transport humans between Earth and other planetary destinations, and to keep them alive on that journey, these systems cannot be launched on a single vehicle from Earth. In-space assembly has the potential to address the challenges of delivering and preparing the necessary systems to satisfy exploration goals. This paper presented a framework of assembly strategies, tasks, and capabilities that facilitate development and assessment of a variety of applications. In particular, potential focus areas for supporting human missions to Mars were identified, holistically considering all mission phases from Earth to Mars, as well as identifying the constraints on the solution space. The focus areas identified were assembly in Earth and/or Mars orbit, assembly on the surface of Mars, assembly to enable or enhance EDL, assembly to enable or enhance the Mars transit vehicle, and assembly to provide communications/nav infrastructure. This framework also allowed for the examination of different ISA approaches based on different assembly strategies, and the determination of how those architectural approaches will impact future human Mars campaigns. Thus, this work provides a foundation for future development and analyses of assembly-based human exploration architectures. These analyses are ongoing and will be reported on in a future publication.

\section{Appendix}

\section{Lexicon of Additional Terms}

Purpose: This appendix captures terminology used within the in-space assembly seedling study, as well as distinctions between how terms are used.

\section{System types associated with assembly}

Mission system - these are the vehicles/spacecraft/elements (or combination thereof) that are being assembled, and that will be conducting the primary mission

Assembly agent - a system that performs the assembly tasks. May be a free-flying spacecraft, arms or other systems supported by infrastructure (habitat, platform, etc.), or manipulators and other devices incorporated on the spacecraft being assembled (e.g. a spacecraft with a robotic manipulator that installs stowed components, e.g. arrays or antennae, once in space).

Infrastructure - facilities in space the support and facilitate assembly. Infrastructure can provide a berth for the spacecraft being assembled; store parts and supplies for both the vehicle and the assembly agents; provide services such as power, thermal, and communications; and host assembly agents.

Note: in some cases, a mission system being assembled may become an assembly agent or infrastructure (e.g. a space dock or assembly platform) for future in-space assembly operations. 
System Nomenclature Hierarchy (as used in this study)

Component Hierarchy

Mission system - An integrated set of one or more elements that accomplish specific mission objectives (e.g. a Mars transit vehicle consisting of a propulsion element and 1 or more habitat elements, a fully assembled space telescope, a fully functional space dock used to support future assembly operations).

Element - An integrated, fully functional set of subsystems and parts that comprise a mission system (e.g. a habitat element, a propulsion element).

Subsystem - An integrated set of smaller assemblies and parts that performs a cleanly and clearly separated function (e.g. ECLSS, power, and communications).

Subassembly - An integrated set of parts that comprise a well-defined portion of a subsystem (e.g. instrument/sensor packages, O2 regeneration in ECLSS, solar panel and boom).

Part - The lowest level of separately identifiable items (e.g. valves, sensors, motors, structural struts, etc.)

Generic Terms.

Assembly - General term for an integrated, well-defined set of parts, subassemblies, or subsystems

Module - General term for partially integrated components (from subassemblies to full elements), intended to be combined with other modules through simplified interfaces to build an integrated, fully functional element.

Component - General term for any level, part through element

\section{Organizational Hierarchy Terms}

Assembly strategy - Operational approaches that represent the differing degrees and assembly and operational complexity that can be employed when assembling a mission system

Assembly task - High-level operations performed in the process of assembling the mission system. The assembly tasks can be sequenced together to create an assembly con-ops. Assembly tasks can be decomposed into constituent functions to identify capability needs and functional requirements for the mission system, assembly agents, and supporting infrastructure.

Function - The lowest level of assembly task decomposition that reflects a capability that must be provided to enable performance of an assembly task

Capability - The ability to perform lower level functions that combine to perform tasks to assemble a mission system. They represent basic functionality needed to perform a given task.

Solution - Design options for providing a capability need. Solutions are the actual hardware and software, and can includes engineering options that are currently available or technologies needing development.

\section{Assembly vs. Manufacture and Fabrication}

Assembly - Assembling a system from existing parts delivered to the assembly location.

Manufacturing - Producing components from basic parts; for example assembling a rotary joint from nuts, bolts, and gears.

Fabrication - Producing basic parts or components from raw materials; for example extruding truss members from feedstock.

\section{$\underline{\text { Automation vs. Autonomy }}$}

Automation - Hardware and software processes that execute step-by-step sequences to perform operations without external control, but that still require human interaction for decision making and fault management. Autonomic systems are provided with inputs to initiate actions.

Autonomy - State where equipment is self-governing and can make decisions and perform operations without human input or guidance. Autonomous systems collect inputs on their own to make decisions and initiate actions. 


\section{References}

${ }^{1}$ Craig, D. A., Troutman, P., Herrmann, N. B., "Pioneering Space Through and Evolvable Mars Campaign," AIAA SPACE 2015,AIAA SPACE Forum, (AIAA 2015-4409)

${ }^{2}$ Human Exploration of Mars Design Reference Architecture 5.0, NASA-SP-2009-566, July 2009.

${ }^{3}$ Musk, E., "Making Humans a Multi-Planetary Species,” New Space. June 2017, 5(2): 46-61. https://doi.org/10.1089/space.2017.29009.emu

${ }^{4}$ Thronson, H., (ed) et al., "The Fourth Community Workshop on Achievability and Sustainability of Human Exploration of Mars," Monrovia, CA, Dec 2016.

${ }^{5}$ Space Launch System Mission Planner's Guide, NASA ESD 30000, April 2017.

${ }^{6}$ Jefferies, S. A., Collins, T., Cianciolo, A. D., Polsgrove, T., "Impacts of Launch Vehicle Fairing Size on Human Exploration Architectures" IEEE Aerospace Conference, Big Sky, MT, 2017.

${ }^{7}$ Watson, J. J., Collins, T. J., and Bush, H. G., "A History of Astronaut Construction of Large Space Structures at NASA Langley Research Center," IEEE Aerospace Conference Proceedings, Vol.7, 2002, pp. 3569-3587.

${ }^{8}$ Belvin, W. K., et al., "In-Space Structural Assembly: Applications and Technology," SCITECH 2016, San Diego, CA, Jan. 2016, AIAA pp 2016-2163.

${ }^{9}$ Hamill, D., Bowman, L. M., Belvin, W. K., and Gilman, D. A., "High Leverage Technologies for In-Space Assembly of Complex Structures," AIAA SPACE 2016, AIAA SPACE Forum, (AIAA 2016-5397).

${ }^{10}$ Dorsey, John T. and Watson, Judith J., "Space Assembly of Large Structural System Architectures,”AIAA SPACE 2016, AIAA SPACE Forum, (AIAA 2016-5481).

${ }^{11}$ Lymer, J., et al., "Commercial Application of In-Space Assembly," AIAA SPACE 2016, AIAA SPACE Forum, (AIAA 2016-5236).

12 Komendera, E. E., Doggett, W. R., Dorsey, J. T., Debus, T. J., Holub, K., and Dougherty, S. P., "Control System Design Implementation and Preliminary Demonstration for a Tendon Actuated Lightweight In-Space MANipulator (TALISMAN)", AIAA SPACE 2015 Conference and Exposition, AIAA SPACE Forum, (AIAA 2015-4628).

13 Percy, T. K., McGuire, M., Polsgrove, T., "In-space transportation for NASA's Evolvable Mars Campaign", AIAA SPACE 2015 Conference and Exposition, AIAA SPACE Forum, (AIAA 2015-4519).

${ }^{14}$ Simon, M. A., Toups, L, Howe, A. S., and Wald, S., "Evolvable Mars Campaign Long Duration Habitation Strategies: Architectural Approaches to Enable Human Exploration Missions", AIAA SPACE 2015 Conference and Exposition, AIAA SPACE Forum, (AIAA 2015-4514).

15 Simon, M. A., et al., "NASA's advanced exploration systems Mars transit habitat refinement point of departure design," IEEE Aerospace Conference, Big Sky, MT, 2017.

${ }^{16}$ Polsgrove, T. P., et al, "Human Mars Lander Design for NASA's Evolvable Mars Campaign," IEEE Aerospace Conference, Big Sky, MT, 2016.

${ }^{17}$ Polsgrove, T., Thomas, H. D., Cianciolo, A., Collins, T., and Samareh, J., "Mission and Design Sensitivities for Human Mars Landers Using Hypersonic Inflatable Aerodynamic Decelerators,” IEEE Aerospace Conference, Big Sky, MT, 2017.

18 Toups, L., Brown, K., and Hoffman, S. J., "Transportation-Driven Mars Surface Operations Supporting an Evolvable Mars Campaign,” IEEE Aerospace Conference, Big Sky, MT, 2015. 